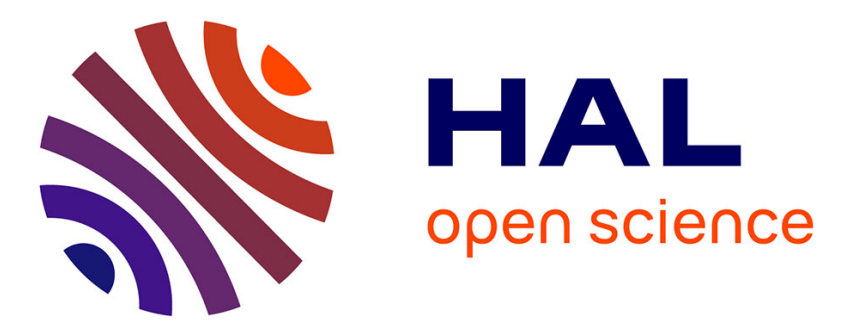

\title{
Relation entre la constante d'anisotropie et la loi d'approche à la saturation des ferromagnétiques
}

Louis Néel

\section{To cite this version:}

Louis Néel. Relation entre la constante d'anisotropie et la loi d'approche à la saturation des ferromagnétiques. J. Phys. Radium, 1948, 9 (6), pp.193-199. 10.1051/jphysrad:0194800906019300 . jpa-00234117

\section{HAL Id: jpa-00234117 https://hal.science/jpa-00234117}

Submitted on 1 Jan 1948

HAL is a multi-disciplinary open access archive for the deposit and dissemination of scientific research documents, whether they are published or not. The documents may come from teaching and research institutions in France or abroad, or from public or private research centers.
L'archive ouverte pluridisciplinaire HAL, est destinée au dépôt et à la diffusion de documents scientifiques de niveau recherche, publiés ou non, émanant des établissements d'enseignement et de recherche français ou étrangers, des laboratoires publics ou privés. 


\title{
LE JOURNAL DE PHYSIQUE
}

\section{RELATION ENTRE LA GONSTANTE D'ANISOTROPIE ET LA LOI D'APPROCHE A LA SATURATION DES FERROMAGNÉTIQUES ( ${ }^{1}$ )}

\author{
Par M. Louis NÉEL.
}

Université de Grenoble, Laboratoire d'Éltctrostatique et de Physique du Métal.

\begin{abstract}
Sommaire. - Quand le champ $H$ croît, l'aimantation đ' d'un ferromagnétique cubique polycristallin tend vers la saturation $\mho_{s}$ selon la loi d'approche

$$
y=y_{s}\left(\mathrm{I}-\frac{b}{H^{2}}\right)
$$

et une théorie actuellement admise montre que $b$ est égal à $8 K^{2}:$ i $05 J_{\zeta}^{2}$ où $K$ est la constante d'anisotropie. L'auteur montre que cette théorie n'est pas correcte car elle a été obtenue en négligeant les interactions magnétiques entre les cristallites dont le rôle est considérable. Les vérifications expérimentales qu'on en a données sont tout à fait illusoires, soit par suite d'interprétations trop sommaires, soit par l'emploi de substances à tensions internes résiduelles trop grandes.

L'auteur développe ensuite une méthode nouvelle de calcul qui permet de tenir compte des interactions magnétiques et obtient ainsi une loi d'approche rigoureuse qui n'a pas la forme (A). Cependant, dans la région pratiquement la plus intéressante, c'est-à-dire de 200 à ı ooo œrsteds, elle peut se mettre approximativement sousla forme $(\mathrm{A})$, mais la valeur de $b$ est à peu près deux fois plus petite que la valeu, donnée plus haut. De nouvelles mesures effectuées sur du nickel bien pur et bien recuit ont confirmé ce résultats. Les formules obtenues permettent de déterminer $K$ au moyen de mesures effectuées sur de
\end{abstract} échantillons polycristallins.

\section{Introduction.}

1. Détermination de la constante $K$ d'anisotropie, par l'étude de l'approche à la saturation. - Cas d'un monocristal. - On sait que l'énergie libre $F$ d'un cristal ferromagnétique est la somme de plusieurs termes dont l'un $F_{m}$ appelé énergie magnétocristalline, dépend de l'orientation de l'aimantation spontanée $\%$ par rapport aux axes cristallins. Dans un cristal cubique, l'expression suivante donne une représentation satisfaisante de $F_{m}$, en fonction des cosinus directeurs $\alpha, \beta, \gamma$, de $q$ par rapport aux axes quaternaires

$$
F_{m}=h\left(\alpha^{2} \gamma^{2}+\beta^{2} \gamma^{2}+\gamma^{2} \alpha^{2}\right) .
$$

(1) Les travaux expérimentaux et théoriques qui font l'objet de cet arti.le ont été poursuivis et achevés pendant l'occupation. La parution en a été retardée par suite de difficultés matérielles. Devant les retards d'impression qui s'annonçaient déjà en 1945 , une brève note a été publiée sur le même sujet (C.R. Acad. Sc., I 945,220, p. 8 I 4 ).
Si le cristal est soumis à un champ l'énergie libre comprend en outre un sece $\cdots, \quad$, " égal à $-\vec{H} \vec{y}$.

Dans un cristal parfait, la position | $1: 1,1, \cdots$ de $\vec{\Im}_{s}$ s'obtient en écrivant q $\imath^{\prime}$ libre $F=F_{m}+F_{h}$ est minimum. champ $H$ devient de plus en plus inter. A, 4. $n$ gardant une direction donnée par, s'ult du cristal, l'aimantation spontanée tend $\rightarrow$ levenir parallèle au champ et sa composant، ." uivant cette direction, tend vers l'aimantat" " :aturation $y_{s}$ On montre facilement qu+ : $\ldots, \ldots$ à la saturation s'effectue suivant une $1 . ., \cdot, t_{,}::_{1}$

$$
\mathfrak{J}=\mathfrak{y}_{2}\left(\mathrm{I}-\frac{b}{\boldsymbol{H}^{2}}\right)
$$

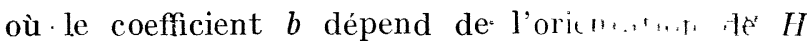
par rapport aux axes du cristal.

LE JOURNAL DE PHYSIQUR ET LE RADIUM. - SÉRIE VIII. - T. IX. - No 6. - JUIX 1948. 
Cas d'un polycristal. - La formule précédente, applicable à un monocristal, n'a fait l'objet d'aucune vérification expérimentale, mais depuis longtemps on s'est proposé de l'appliquer à un polycristal. Le premier, Akulov ( ${ }^{2}$ ) a calculé la valeur de $b$ relative à un polycristal en faisant la moyenne des valeurs obtenues en donnant à un monocristal toutes les orientations possibles; on obtient ainsi

$$
b=\frac{8}{10 \check{J}} \frac{K^{2}}{J_{\frac{2}{3}}^{\frac{2}{3}}} .
$$

Gans $\left({ }^{3}\right)$ et plus tard Becker $\left(^{4}\right)$ ont repris le même raisonnement et obtenu le même résultat.

Vérifications expérimentales. - Entre temps, Czerlinski ( $\left.{ }^{5}\right)$ a montré expérimentalement que les polycristaux de fer et de nickel obéisssaient à une loi d'approche de la forme (2) et, à partir des valeurs de $b$ ainsi obtenues et compte tenu de la formule (3), il en a déduit des valeurs de $K$ qui, entre - $150^{\circ}$ et $150^{\circ} \mathrm{C}$, concordent avec les valeurs de la littérature déterminées par les méthodes habituelles applicables aux monocristaux. De son côté, Polley ( ${ }^{6}$ ) a repris l'étude du polycristal de nickel et montré qu'en réalité il fallait remplacer la loi d'approche (2) par une loi plus complexe de la forme

$$
\mathfrak{J}=\mathfrak{J}_{s}\left(\mathrm{I}-\frac{a}{H}-\frac{b}{H^{2}}\right)+\chi H ;
$$

mais, en supposant que le terme en $\frac{a}{H}$ provenait d'un mécanisme inconnu mais n'altérant pas le terme $\frac{b}{H^{2}}$, il a pu, comme plus haut, calculer à d.fférentes températures des valeurs de $K$ qui s'accordent encore avec celles de la littérature.

1) semble donc que nous soyons en possession d'une méthode éprouvée de détermination des coeflicients d'anisotropie $K$, à partir de mesures effectuées sur des polycristaux, ce qui présente un intérít expérimental évident. Malheureusement, on peut élever contre cet ensemble de résultats de graves objections, tant du point de vue théorique que du point de vue expérimental.

2. Objections d'ordre théorique à la méthode précédente. - Il ne semble pas légitime de calculer le coefficient $b$ relatif à un polycristal en prenant la moyenn des valeurs de $b$ relatives aux cristallites élémentair's supposés isolés, car on néglige ce faisant les champs démagnétisants internes développés par les charges magnétiques qui proviennent de la non-.iniformité de l'aimantation du polycristal.

(2) Anulore. Physik, r $931, \quad 69, \quad$ p. 822

(3) Gis, der Physik, 1932, 15, p. 28.

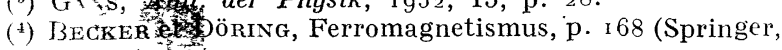
Berlin, 1939 ).

(5) Czerlisski, Ann. der Physik, 1932, 13, p. 80.

(6) $\mathrm{PO}$ inn. der Physik, r939, 36, p. 625.
Pour un cristallite isolé, la présence du champ démagnétisant n'introduit pas de difficulté spéciale : le cristallite est en moyenne sphérique de sorte que son coefficient moyen de champ démagnétisant est indépendant de la direction et égal à $\frac{4}{3} \pi$. D'autre part, comme là théorie suppose implicitement un champ appliqué suffisamment intense pour qu'il n'existe qu'un seul domaine élémentaire par cristallite, il en résulte que l'aimantation d'un cristallite est toujours égale à l'aimantation spontanée et que les seuls changements d'aimantation à considérer proviennent de la pure rotation d'un vecteur de grandeur $J_{s}$ invariable $\left({ }^{7}\right)$, rotation au cours de laquelle l'énergie de champ démagnétisant reste invariable et égale à $\frac{1}{2} \frac{4}{3} \pi_{s} J_{s}^{2} \operatorname{ergs~}: \mathrm{cm}^{3}$. Il n'y a donc pas à tenir compte de ce champ démagnétisant.

Il n'en est plus de même pour l'ensemble des cristallites juxtaposés qui constitue le polycristal. Supposons en effet que les cristallites possèdent ainsi réunis, le même état d'aimantation qu'à l'état isolé. Comme la direction de l'aimantation spontanée varie d'un cristallite à l'autre, il apparaît sur les surfaces de séparation des charges magnétiques fictives qui créent des champs magnétiques et augmentent ainsi l'énergie libre. Mais, tandis que dans un cristallite isolé cette énergie conserve la même valeur lorsque l'aimantation devient parallèle au champ appliqué, ici au contraire cette énergie supplémentaire disparaît à la saturation lorsque tous les cristallites prennent des aimantations parallèles (on suppose que le polycristal a la forme d'un barreau allongé dans la direction du champ). Il en résulte nécessairement des forces qui tendent à faciliter la saturation.

On peut apprécier l'effet de ces interactions par le raisonnement suivant : si l'on extrait d'un polycristal un cristallite élémentaire, le champ qui règne dans la cavité correspondante est, en moyenne égal à $H+\frac{4}{3} \tau \mathcal{J}$, en désignant par $\mathfrak{J}$ l'aimantation moyenne du polycristal. C'est ce champ total qui agit effectivement sur le cristallite. Comme au voisinage de la saturation, I est pratiquement égal à $J_{*}$, il faut donc remplacer le terme en $\frac{b}{H^{2}}$ de la loi (2) par un terme en $\frac{b}{\left(H+\frac{4}{3} \pi J_{s}\right)^{2}} \cdot$ Dans le cas du nickel, où ¿s est voisin de 500 C. G. S., et dans un champ de rooo orsteds, on constate que le nouveau terme est neuf fois plus petit que le terme primitif. Sans doute le raisonnement esquissé ici est-il trop grossier pour nous donner un résultat

( $\left.{ }^{7}\right)$ Pour cette question, se reporter au mémoire de NÉEL, Les lois de l'aimantation et de la subdivision en domaines élémentaires d'un monocristal de fer, $J$. de Physique, 1944, t. 5, p. $241-251$. 
précis, mais il suffit à montrer que les interactions entre cristallites sont considérables et qu'il ne peut être question de les négliger.

Finalement la loi (2) avec la valeur de $b$ donnée en (3) est grossièrement inexacte.

3. Objections d'ordre expérimental. - Il s'agit alors d'expliquer dans quelles conditions les auteurs précités ont pu obtenir des valeurs correctes de $K$ à partir de formules inexactes.

Expériences de Czerlinski. - En ce qui concerne ces expériences, une discussion serrée du mémoire de l'auteur, qu'il n'y a pas intérêt à reproduire ici, montre que ses résultats sont beaucoup mieux représentés par une formule du type

$$
\mathcal{I}=\mathcal{J}_{s}\left(\mathrm{I}-\frac{a}{H}-\frac{c}{H^{3}}\right),
$$

sans terme en $\frac{b}{H^{2}}$, que par la formule qu'il indique, ne comprenant pas de terme en ${ }_{\bar{H}}$, mais avec un terme en $\frac{b}{H^{2}}$, et qui présente des déviations systématiques par rapport à l'expérience. On obtient d'ailleurs selon cette nouvelle interprétation une valeur de $a$ égale à 5,2 qui est du même ordre de grandeur que les valeurs obtenues par d'autres auteurs pour du fer pur $\left({ }^{8}\right)$. Ainsi les expériences de Czerlinski, loin d'appuyer la théorie d'Akulov-Gans-Becker, montrent plutôt que la valeur du terme en $\frac{b}{H^{2}}$ prévue par celle-ci est trop grande. Il faut cependant remarquer que la présence d'un terme supplémen-. taire en $\frac{a}{\boldsymbol{H}}$, dû probablement aux imperfections des cristallites $\left({ }^{8}\right)$, rend l'interprétation incertaine car il est à craindre que ces imperfections ne se manifestent aussi par un terme supplémentaire en $\frac{b^{\prime}}{H^{2}}$.

Expériences de Polley. - De ce point de vue, les expériences de Polley sur le nickel se présentent plus favorablement, car le terme en $\frac{a}{H} \mathrm{y}$ est très faible, mais l'échantillon de cet auteur doit néanmoins être encore assez éloigné de la perfection, comme le montre la méthode suivante.

4. Signification et calcul du travail d'aimantation. - Dans l'état actuel de nos connaissances, nous nè savons pas calculer la courbe d'aimantation d'un polycristal, mais il existe une grandeur dont la détermination expérimentale est facile et qui,

(8) NéEL, La loi d'approche en i : H, J. de Physique, 1948, 9 , p. 184 . indépendamment de toute théorie, peut être calculée en fonction de la constante d'anisotropie : il s'agit du travail d'aimantation.

Du point de vue expérimental, le travail d'aimantation est égal à l'aire comprise entre la courbe d'aimantation (aimantation en fonction du champ), l'asymptote à la saturation et l'axe des ordonnées. Cette méthode suppose qu'il n'y a pas d'hystérésis; on peut cependant encore, comme l'a montré Kersten $\left({ }^{9}\right)$ dans un cas analogue, évaluer le travail réversible d'aimantation quand l'hystérésis est faible : en mesurant l'aire comprise entre la branche descendante du cycle limite et l'asymptote à la saturation (fig. I) car cette branche SR du cycle est sensiblement réversible.

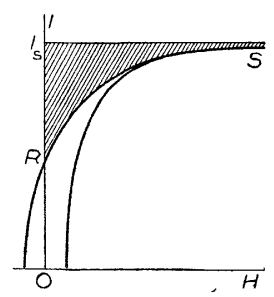

Fig. I. - La branche descendante SR du cycle limite est sensiblement réversible : l'aire hachurée correspond donc au travail d'aimantation.

Du point de vue théorique, le travail d'aimantation est égal à la différence entre l'énergie interne à la saturation et l'énergie interne dans l'état initial, quand l'aimantation est nulle.

Dans cet état initial, l'aimantation spontanée des différents domaines élémentaires est dirigée suivant l'une des directions dites privilégiées: correspondant à un minimum de l'énergie magnétocristalline. Lorsque $K$ est positif (cas du fer), le minimum de l'énergie magnétocristalline $F_{m}$ est zéro; lorsque $K$ est négatif (cas du nickel) ce minimum est égal à $\begin{gathered}K \\ 3\end{gathered}$. D'autre part, à la saturation, l'aimantation spontanée est parallèle au champ appliqué, de sorte que la valeur moyenne de l'énergie d'un polycristal est égale à la valeur moyenne de $F_{m}$ donnée par l'expression (1), quand le vecteur unitaire $\alpha, \beta, \gamma$ prend toutes les orientations possibles : un calcul élémentaire montre que cette moyenne est égale à $\frac{K}{5}$.

Finalement, il en résulte que le travail d'aimantation d'un polycristal est égal à $\frac{K}{5} \mathrm{ergs}: \mathrm{cm}^{3}$, quand $K$ est positif, et égal à $\frac{K}{5}-\frac{h}{3}=\frac{-2 K}{15}$ ergs $: \mathrm{cm}^{3}$, quand $K$ est négatif.

(9) Kersten, Z. Physik, r 932,76, p. 505. 
Application aux expériences de Polley. - Or, d'après les données de la littérature relatives aux monocristaux de nickel ( $c f$, Becker, Loc. cit., p. 123), $K$ est voisin de -5 ,o. I $\mathrm{o}^{4} \mathrm{ergs}: \mathrm{cm}^{3}$, d'où il résulte qu'à la température ordinaire, le travail d'aimantation d'un polycristal de nickel est voisin de $6,6$. I $^{3}$ ergs : $\mathrm{cm}^{3}$.

D'autre part, d'après les données numériques et les graphiques du mémoire de Polley, il résulte qu'à $14^{\circ} \mathrm{C}$ le travail d'aimantation du nickel qu'il a étudié était voisin de $13 . \mathrm{ro}^{3} \mathrm{ergs}: \mathrm{cm}^{3}$, valeur double de la valeur théorique. Malgré le recuit qu'il avait subi, ce nickel était donc probablement affecté de tensions internes relativement considérables altérant de façon importante la courbe a'aimantation; on sait en effet que, par suite de la grandeur de sa magnétostriction, le nickel est particulièrement sensible aux tensions internes.

5. Nouvelles mesures sur le nickel. - Dans ces conditions, il était intéressant de reprendre la mesure du coefficient $b$ sur du nickel pur, débarrassé de tensions internes.

- Méthode. - A cet effet nous avons étudié, à la température ordinaire, un fil de nickel pur. Le fil, de $0,4 \mathrm{~mm}$ de diamètre et de $\mathrm{r} 30 \mathrm{~cm}$ de longueur, était tendu à l'intérieur d'un solénoïde de $950 \mathrm{~cm}$ de longueur. La bobine de flux, convenablement compensée de manière à indiquer directement au galvanomètre balistique les. variations de $4 \pi J$, avait $20 \mathrm{~cm}$ de longueur. Toutes dispositions étaient prises pour pouvoir tracer des cycles, point par point, à partir d'une origine arbitrairement choisie.

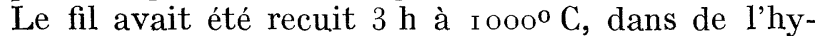
drogène soigneusement purifié. Des recuits ultérieurs, poussés jusqu'à $\mathrm{I} 200^{\circ} \mathrm{C}, \mathrm{n}$ 'ont pas modifié de manière sensible la forme de la courbe d'aimantation.

Résultats. - Ils sont donnés par les figures 2 et 3 . Le champ coercitif de ce nickel était très faible : égal à o,66 œersted. On pouvait donc espérer qu'il était bien libre de tensions internes. Nous avons en effet trouvé que l'aire comprise entre la branche descendante du cycle limite et son asymptote était égale à $6,4 \cdot \mathrm{r}^{3}$ ergs : $\mathrm{cm}^{3}$, à la température de $27^{\circ} \mathrm{C}$; cette valeur est extrêmement voisine de la valeur théorique obtenue plus haut.

Pour préciser la loi d'approche à la saturation du nickel ainsi traité, nous avons sur la figure 3 représenté l'aimantation en fonction de $\frac{\mathrm{I}}{\mathrm{H}^{2}}$. Entre $\mathrm{x}$ รัo et 750 orsteds, on obtient ainsi sensiblement une droite qui s'extrapole vers une aimantation à saturation égale à 497,5 . Dans cet intervalle de champ, les termes en $\stackrel{a}{\vec{H}}$ et en $\% H$ du développement (4) ne sont pas sensibles. La formule (2) suffit alors pour représenter les expériences avec un coefficient $b$ égal à 290 , à la température de $27^{\circ} \mathrm{C}$. Pour cette même température, en interpolant les résultats de Polley, on obtiendrait $b=550$, valeur sensiblement double de la précédente.

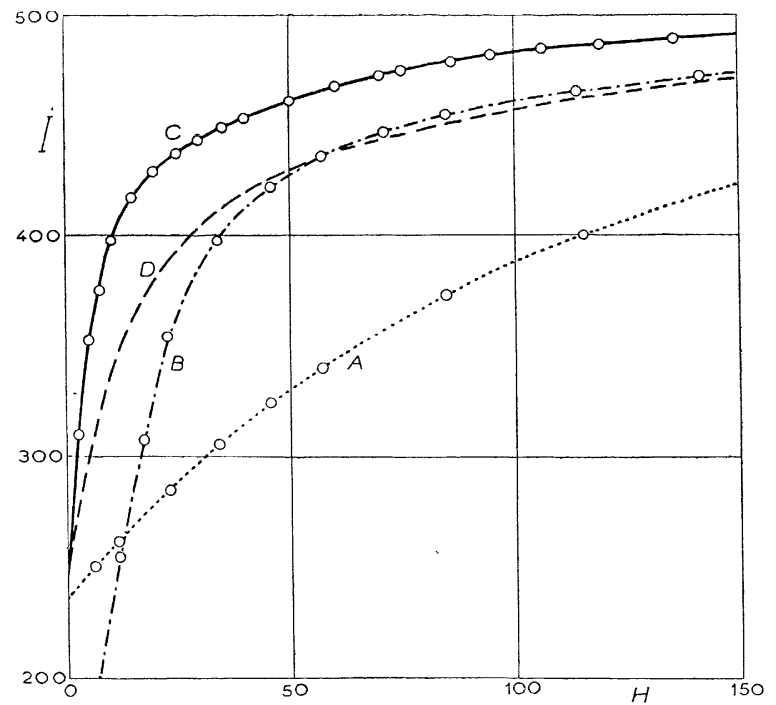

Fig. 2. - Courbes d'aimantation dans des champs moyens de différents échantillons de nickel : $A$, fil tel qu'il sort de la bobine $\left(H_{c}=8,7 œ\right) ; \mathrm{B}$, le même fil recuit $\mathrm{I} 5 \mathrm{~m}$ à $900^{\circ}$ dans l'air $\left(H_{c}=5,4 œ\right)$; C, le même recuit $3 \mathrm{~h}$ à I $000^{\circ}$ dans l'hydrogène $\left(H_{c}=0,7 œ\right) ; \mathrm{D}$, fil étudié par Polley.

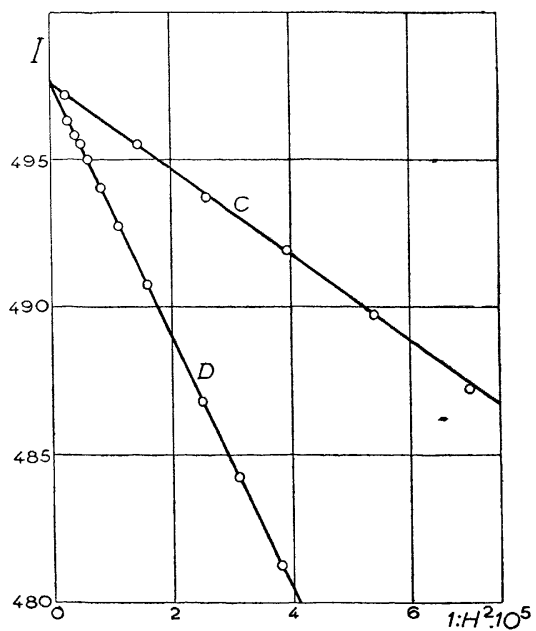

Fig. 3. - Approche à la saturation dans les champs forts, en fonction de l'inverse du carré du champ : C, fil de nickel recuit $3 \mathrm{~h}$ à $1000^{\circ}$ dans l'hydrogène; D, fil étudié par Polley (d'après ses mesures).

Cette différence entre les propriétés de deux échantillons de nickel pur ayant subi des traitements thermiques voisins est difficiie à expliquer : remarquons simplement que l'échantillon de Polley était plus massif que le nôtre, ayant $5 \mathrm{~mm}$ de 
diamètre, et que les impuretés pouvaient y avoir plus de difficultés pour diffuser à la surface et s'éliminer; en outre, Polley ne semble pas avoir pris de précautions spéciales pour purifier l'hydrogène.

Quoi qu'il en soit, il en résulte que du point de vue expérimental, pour du nickel aussi bien débarrassé que possible des tensions parasites, le terme en $\frac{b}{\boldsymbol{H}^{2}}$ de la loi d'approche est, entre 200 et 700 œsteds, deux fois plus petit que le terme théorique, calculé en négligeant les interactions entre les cristallites.

\section{Théorie de la loi d'approche d'un polycristal.}

6. Représentation analytique des forces magnétocristallines dans un polycristal. Exposons maintenant une méthode de calcul applicable à un polycristal constitué de cristallites élémentaires, dont les orientations sont réparties au hasard, placé dans un champ magnétique suffisant pour qu'en tout point l'aimantation soit voisine de la saturation. En chaque point, l'aimantation est en équilibre sous l'action du champ magnétique appliqué $H$ et des forces magnétocristallines. Tant qu'on se borne à étudier l'approche à la saturation, ces dernières forces sont équivalentes du point de vue formel à un champ magnétique $F$, d'intensité convenable, perpendiculaire au champ $H$ : naturellement la grandeur et l'orientation de $F$ changent d'un cristallite à l'autre.

Découpons d'autre part dans le polycristal un cube de côté $L$, très grand vis-à-vis des dimensions des cristallites. Il est clair qu'en juxtaposant les uns aux autres, par des translations suivant trois directions rectangulaires, des cubes identiques au premier, on obtient un polycristal périodique indéfini de propriétés pratiquement identiques à celles du polycristal initial. Ceci posé, rapportons ce polycristal périodique à trois axes rectangulaires $\mathrm{OXYZ}$. La composante du champ $F$ suivant une direction $\Delta$ quelconque est développable en une série de Fourier

$$
F_{\delta}=\sum M_{p q r} \exp \left\{\frac{2 i \pi}{L}(p x+q y+r z)\right\}
$$

où le signe somme est étendu, ici comme dans tout ce qui va suivre, à toutes les valeurs entières de $p q r$ de $-\infty$ à $+\infty$. Les coefficients $M_{p q r}$ sont des quantités complexes qui, $F_{j}$ étant réel, satisfont à la relation

$$
M_{-p, m q,-r}=M_{p \eta r}^{\star}
$$

en désignant par un astérisque * l'imaginaire conjuguée.

En découpant le cube initial dans un autre endroit du polycristal, on obtient naturellement des coef- ficients $M_{\mu, y}$. entièrement différents de sorte qu'il importe plutôt de connaître leurs valeurs probables que leurs valeurs réelles pour un développement particulier. Comme le polycristal est en moyenne isotrope, il en résulte que, comme nous l'avons déjà montré sur un exemple analogue $\left({ }^{8}\right)$, la loi de probabilité qui fixe la valeur de $M_{p q}$. ne dépend que du seul paramètre : $p=1 \overline{p^{2}+q^{2}+r^{2}}$, car le développement doit conserver la même forme pour une rotation quelconque des axes. En particulier, la valeur moyenne $\overline{M_{p q r} M^{*}{ }_{p q r}}$ du produit $M_{p q r} M^{*}{ }_{p q r}$ est une fonction positive de $\rho$.

Supposons alors les axes orientés de manière à ce que OZ soit parallèle au champ appliqué $H$; les composantes $F_{x}$ et $F_{y}$ de $F$ suivant $\mathrm{OX}$ et $\mathrm{OY}$ sont représentables par deux développements du type (6), identiques en moyenne par raison de symétrie. Les coefficients correspondants, $M_{p q r}$ et $M_{p q r}^{\prime}$ obéissent aux relations suivantes, où l'on a pris la valeur moyenne, par rapport à toutes les positions possibles du cube initial, de la quantité située au-dessous du trait

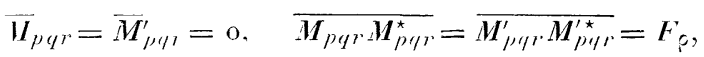

où $F_{p}$ est une quantité positive qui ne dépend que de $p$. En outre, puisque les $M$ et les $M^{\prime}$ sont indépendants, on a

$$
\overline{\boldsymbol{M}_{p q r} \boldsymbol{M}_{p q r}^{\prime \star}}=\mathrm{o}, \quad \overline{\boldsymbol{M}_{p q r}^{\star} \boldsymbol{M}_{p q r}^{\prime}}=\mathrm{o} .
$$

7. Loi d'approche sans interactions entre les cristallites. - Désignons par $\alpha$ et $\beta$ les angles avec OZ des projections sur les plans XOZ et YOZ de l'aimantation $\nearrow_{s}$ en un point. Puisque les angles $\alpha$ et $\beta$ sont petits, la conditions d'équilibre de l'aimantation s'écrit simplement

$$
\alpha=\frac{F_{x}}{H}, \quad \beta=\frac{F_{r}}{H} ;
$$

et, au second ordre près, l'aimantation macroscopique $\mathscr{J}$, égale à la valeur moyenne de la projection de l'aimantation sur OZ, s'écrit

$$
\mathfrak{y}=\dot{\jmath}\left(\mathrm{I}-\frac{\bar{\alpha}^{2}+\bar{\gamma}^{2}}{2}\right)=J_{s}\left(\mathrm{I}-\frac{\bar{F}_{\bar{x}}^{2}+\bar{F}_{y}^{2}}{2 H^{2}}\right) .
$$

Pour obtenir $\overline{F_{x}^{2}}$, il faut, après avoir élevé l'expression (6) au carré, effectuer successivement les moyennes par rapport à $x y z$ et par rapport à toutes les valeurs possibles des coefficients $M$; on obtient finalement, compte tenu des relations (7) et (8)

$$
\overline{F_{i}^{*}}=\bar{F}_{1}^{2}=\sum \overline{M_{p q r} M_{p q r}^{*}}=\sum F_{p}
$$

d'où la loi d'approche

$$
J=\mathcal{J}_{S}\left(\mathrm{I}-\frac{\Sigma F_{\mathrm{P}}}{H^{q}}\right),
$$


Comme cette loi doit être identique à la loi donnée par les méthodes classiques, on déduit, après avoir rapproché les relations (2), (3) et (13)

$$
\sum F_{\rho}=\frac{8}{\text { IOว̆ }} \frac{K^{2}}{J_{*}^{2}} \text {. }
$$

8. Introduction des interactions. - Nous allons maintenant introduire les interactions entre les cristallites qui avaient été négligées par.Akulov et ses successeurs : Gans et Becker. Ces interactions se manifestent par l'existence d'un champ démagnétisant interne dérivant d'un potentiel $V$ et provenant de la non-uniformité de l'aimantation du polycristal. La condition d'équilibre (10) doit être remplacée par la suivante :

$$
\alpha=\frac{\mathrm{I}}{\boldsymbol{H}}\left(F_{x}-\frac{\partial V}{\partial x}\right) ; \quad \beta=\frac{\mathrm{I}}{\boldsymbol{H}}\left(F_{y}-\frac{\partial V}{\partial y}\right) .
$$

D'autre part, le potentiel $V$ satisfait à l'équation de Poisson : $\Delta V+4 \pi m=0$ avec $m=-$ div $\mathcal{J}$ Mais comme les composantes locales de l'aimantation sur les trois axes sont, au second ordre près, respectivement égale à $\alpha \mathscr{J}_{s}, \beta \mathscr{I}_{s}$ et $\mathscr{J}_{s}$ on en déduit

$$
\begin{aligned}
& -m=\frac{\partial \mathscr{J}_{x}}{\partial x}+\frac{\partial \mathscr{J}_{x}}{\partial y}+\frac{\partial \mathscr{J}_{z}}{\partial z} \\
= & \frac{\mathscr{J}_{s}}{H}\left(\frac{\partial F}{\partial x}+\frac{\partial F^{\prime}}{\partial y}-\frac{\partial^{2} V}{\partial x^{2}}-\frac{\partial^{2} V}{\partial y^{2}}\right)
\end{aligned}
$$

et l'équation de Poisson prend la forme

$$
\begin{gathered}
\left(\mathrm{I}+\frac{4 \pi J_{s}}{H}\right)\left(\frac{\partial^{2} V}{\partial x^{2}}+\frac{\partial^{2} V}{\partial y^{2}}\right)+\frac{\partial^{2} V}{\partial z^{2}} \\
=\frac{4 \pi J_{s}}{H}\left(\frac{\partial F}{\partial x}+\frac{\partial F}{\partial y}\right) .
\end{gathered}
$$

Des développements du type (6) qui définissent $F_{x}$ et $F_{y}$, on déduit

$$
\begin{aligned}
\frac{\partial F}{\partial x}+\frac{\partial F}{\partial y}= & \frac{2 i \pi}{L} \sum\left(p M_{p q r}+q M_{p q r}^{\prime}\right) \\
& \times \exp \left\{\frac{2 i \pi}{L}(p x+q y+r z)\right\} \\
\bar{\alpha}^{2}+\overline{\bar{\beta}}^{2} & =\frac{1}{H^{2}} \int_{-\infty}^{+\infty} \int_{-\infty}^{+\infty} \int_{-\infty}^{+\infty} F_{P}\left\{\mathrm{I}+\left[\frac{18}{\rho^{2}}\right.\right.
\end{aligned}
$$

L'équation (17) est donc satisfaite par un développement de la forme

$$
V=\sum v_{p q r} \exp \left\{\frac{2 i \pi}{L}(p x+q y+r z)\right\},
$$

où les coefficients $v$ sont donnés par

$$
\left.\begin{array}{c}
v_{p q r}=\frac{b L\left(p M_{p q r}+q M_{p, r}^{\prime}\right)}{2 i \pi\left[b\left(p^{2}+q^{2}\right)+p^{2}+q^{2}+r^{2}\right]} \\
\text { avec } \quad b=\frac{4 \pi J_{s}}{M} .
\end{array}\right\}
$$

En reportant cette expression de $V$ dans la relation (15), on en déduit comme valeur moyenne de $\alpha^{2}$ par rapport à $x y z$

$$
\begin{aligned}
\bar{\alpha}^{2}=\frac{\mathrm{I}}{H^{2}} & \sum\left[\frac{b p\left(p \boldsymbol{M}+q \boldsymbol{M}^{\prime}\right)}{b\left(p^{2}+q^{2}\right)+p^{2}}-\boldsymbol{M}\right] \\
& \times\left[\frac{b p\left(p M^{\star}+q M^{\star \star}\right)}{b\left(p^{2}+q^{2}\right)+p^{2}}-M^{\star}\right]
\end{aligned}
$$

et une expression symétrique pour $\overrightarrow{\beta^{2}}$.

En effectuant ensuite la moyenne par rapport à toutes les valeurs possibles des $M$ et $\operatorname{des} M^{\prime}$ et compte tenu des relations (8) et (9), on obtient comme valeur moyenne $\overline{\alpha^{2}}+\overline{\beta^{2}}$ de $\overline{\alpha^{2}}+\overline{\beta^{2}}$

$$
\overline{\alpha^{2}}+\overline{\beta^{2}}=\frac{1}{H^{2}} \sum F_{\rho}\left\{1+\left[\frac{\rho^{2}}{\rho^{2}+b\left(p^{2}+q^{2}\right)}\right]^{2}\right\} .
$$

Comme le nombre des termes compris sous le signe somme est très grand et que la probabilité d'en rencontrer un dans le volume $\mathrm{d} p \cdot \mathrm{d} q \mathrm{~d} r$ est partout simplement égale à $\mathrm{d} p \mathrm{~d} q \mathrm{~d} r$, on peut remplacer le signe somme par une intégrale et en effectuant le changement de variable : $p=\rho \sin \varphi \cos \theta, \quad q=\rho \sin \varphi \sin \theta, \quad r=\rho \cos \varphi$, $\mathrm{d} p \mathrm{~d} q \mathrm{~d} r=\rho^{2} \sin \varphi \mathrm{d} \varphi \mathrm{d} \rho \mathrm{d} 0$, on constate que les variables se séparent et en intégrant par rapport à $\theta$, il vient où $G$ est l'intégrale définie

$$
G=\int_{0}^{\frac{\pi}{2}}\left[\mathrm{I}+\frac{\mathrm{I}}{\left(\mathrm{I}+b \sin ^{2} \varphi\right)^{2}}\right] \sin \varphi \mathrm{d} \varphi,
$$

qu'un calcul élémentaire donne égale à

$$
\begin{aligned}
G=\mathrm{I} & +\frac{\mathrm{I}}{2(b+\mathrm{I})} \\
& +\frac{\mathrm{I}}{2(b+\mathrm{I})^{2}} \sqrt{\frac{b+\mathrm{I}}{b}} \operatorname{argth} \sqrt{\frac{b}{b+\mathrm{I}}} .
\end{aligned}
$$

D'autre part, la même transformation de somme en intégrale appliquée à la somme $\Sigma F_{q}$ donne

$$
\sum F_{p}=4 \pi \int_{0}^{\infty} \rho^{2} F_{p} \mathrm{~d} p \text {. }
$$

Finalement en substituant l'expression (23) dans l'équation (11) et compte tenu des relations (26) et (14), on aboutit à la loi d'approche suivante où $G$ est donné par l'équation (25) avec $b$ égal à $\frac{4 \pi J_{s}}{\boldsymbol{H}}$

$$
\mathscr{I}=\mathscr{J}_{\mathcal{S}}\left(\mathrm{I}-\frac{8 K^{2}}{\mathrm{I} 05 \mathfrak{J}_{s}^{2} H^{2}} \frac{G}{2}\right)
$$

9. Discussion de la loi d'approche (27). Supposons d'abord que le champ appliqué soit très grand; dans ce cas $b$ est très petit et l'on trouve qu'en négligeant les termes du second ordre en $b, \frac{G}{2}$ se 
réduit à $\frac{1}{\left(1+\frac{b}{3}\right)^{2}} \cdot$ La loi (27) devient alors

$$
\mathscr{J}=\mathscr{J}_{s}\left[\mathrm{I}-\frac{8 \boldsymbol{K}^{2}}{10 \mathfrak{J}_{\mathfrak{s}}^{2}\left(H+\frac{4}{3} \pi J_{s}\right)^{2}}\right] .
$$

On constate alors que les interactions entre les cristallites ont pour effet d'ajouter au champ appliqué un champ égal à $\frac{4}{3} \pi \mathfrak{Y}_{s}$; on reconnaît là le terme classique de Lorentz qu'un raisonnement sommaire nous avait donné plus haut. Mais, du point de vue pratique, il est impossible de se placer dans des conditions où la formule (28) soit valable : en effet, comme pour le fer $4 \pi \mathcal{J}_{s}$ est déjà égal à 2 I 500 , il faut atteindre roo ooo œrsteds pour que $b$, à la rigueur, puisse être considéré comme petit. On sait au contraire que, dans l'étude expérimentale de la loi d'approche en $\frac{\mathrm{I}}{\boldsymbol{H}^{2}}$, les champs utilisés re dépassent pas 2000 œrsteds. Dans ces conditions, $b$ doit plutôt être considéré comme grand. Le calcul numérique de l'expression (25) montre que $G: 2$, égal à 0,5 quand $\frac{\mathrm{I}}{b}$ est nul, croît à peu près linéairement avec $\frac{\mathrm{r}}{b}$ jusqu'à $\frac{\mathrm{I}}{b}=0,3$. C'est ainsi qu'on obtient les chiffres du tableau suivant :

\begin{tabular}{lllll}
\multicolumn{5}{c}{ Tableat. } \\
$\frac{\mathrm{I}}{b} \cdots$ & 0,022 & 0,050 & $0, \mathrm{II} 6$ & 0,276 \\
$\frac{G}{2} \cdots$ & 0,506 & $0,5 \mathrm{r} 3$ & 0,531 & 0,576
\end{tabular}

en se limitant au domaine accessible à l'expérience. Dans cet intervalle, $G$ varie assez peu avec $H$ pour que la loi d'approche réelle puisse être confondue en première approximation avec une loi rigoureuse en $\frac{\mathrm{I}}{\boldsymbol{H}^{2}}$, conformément à l'expérience, mais le coefficient du terme en $\frac{1}{H^{2}}$ est sensiblement deux fois plus petit que celui que l'on obtient en négligeant les interactions.

Dans les mesures sur un fil polycristallin de nickei qui ont été exposées précédemment (\$ 5), le domaine d'étude de la loi d'approche en $\frac{\mathrm{I}}{\boldsymbol{H}^{2}}$ s'étendait de $H=$ г ว̆o à $H=.750$ orsteds $: \frac{\mathrm{I}}{b}$ variait donc de 0,024 à $0, \mathrm{I}$ et la valeur moyenne correspondante de $\frac{G}{2}$, d'après le tableau, était d'environ o,5 г 5 . Le coefficient du terme en $\frac{\mathrm{I}}{H^{2}}$ ayant été trouvé égal à 29o, on en déduit d'après la formule (27), $K=1,3 . \mathrm{Io}^{4}$ ergs $: \mathrm{cm}^{3}$, pour $t=27^{\circ} \mathrm{C}$. A cette même température, les mesures de Brukhatov et Kirensky (10) sur un monocristal donnent $K=4.16$. I $^{4}$ ergs: $\mathrm{cm}^{3}$. L'accord entre ces deux valeurs est excellent.

10. Conclusions. - Ainsi, en ce qui concerne le nickel, l'introduction des interactions entre les cristallites conduit à une formule qui s'accorde avec les résultats expérimentaux.

Inversement, il semble qu'on puisse utiliser la formule (27) pour déduire de la loi d'approche la constante d'anisotropie $K$; à titre de première approximation, on pourra poser $G=\mathbf{r}$, c'est-à-dire utiliser la formule d'Akulov-Becker, mais avec un coefficient deux fois plus petit.

On peut également déduire $K$ de la mesure de l'énergie d'aimantation, en suivant la méthode exposée dans le paragraphe 4 , mais cette méthode paraît moins sûre et moins commode.

D'une manière générale, il sera bon de s'assurer, en utilisant les rayons $\mathrm{X}$ par exemple, que la substance est réellement isotrope et que les axes cristallins sont effectivement répartis au hasard, ce qui n'est pas toujours le cas des fils étirés. Il faut, en outre, que la substance soit aussi bien débarrassée que possible de tensions internes et ne contienne ni cavités ni inclusions non magnétiques : en effet, comme nous l'avons montré antérieurement $\left(^{8}\right)$, la présence des cavités perturbe profondément la loi d'approche. Ces précautions étant prises, il semble qu'on puisse tirer un bon parti des mesures sur les polycristaux, dans l'étude de l'anisotropie magnétique des substances cubiques.

(10) Brukhatov et Kirensky, Physik $Z$. Sowjetunion, 1937 12, p. 602 . 